\title{
Peer Victimization and Emotional Problems in Vietnamese Children: A Longitudinal Study
}

\author{
Tuan Ba Vu ${ }^{1} *$, Katja Petry ${ }^{2}$, Guy Bosmans ${ }^{3}$
}

\section{ABSTRACT}

This study aimed to test the hypothesis that peer victimization in middle childhood predicts emotional problems in adolescents. The sample consisted of 1,000 students administrated at age 8 (wave 1) who were followed for seven years (wave 2). The peer victimization measures included one item derived from Goodman's Strength and Difficulties Questionnaire (SDQ) (wave 1) and nine other items developed by Young Lives' researchers (wave 2). The emotional problems measure used in both waves is SDQ's Emotional Symptoms Subscale. Primary caregivers reported these measures at wave 1 and the children themselves reported at wave 2 . Results revealed that current emotional problems significantly mediate the link between peer victimization and later emotional problems.

Keywords: Peer Victimization, Emotional Problems, Longitudinal Study, Cross-Sectional Study, Emotional Symptoms

Peer victimization is the experience that children undergo when they are chronically victimized by other children's aggressive behavior, such as bullying, teasing or harassment, at or out of school (Hawker \& Boulton, 2000; Goodman et al, 2001). Evidence suggests that peer victimization is associated with emotional problems in both cross-sectional and longitudinal relationships. Regarding the former, for instance, in a meta-analytic review of cross-sectional studies, Hawker \& Boulton (2000) indicated that peer victimization positively correlated with some forms of emotional problems such as depression and anxiety. Regarding the latter, peer victimization also has long-term negative effects on children's emotions (Reijntjes et al., 2010). More specifically, peer victimization causes some forms of emotional problems longitudinally, from mild forms such as emotional symptoms manifested in a one-year follow-up (Malti et al., 2009), to severe forms such as anxiety and depression symptoms manifested two years later

\footnotetext{
${ }^{1}$ PhD candidate, Faculty of Psychology and Educational Sciences, KU Leuven, Belgium

${ }^{2}$ Professor, Faculty of Psychology and Educational Sciences, KU Leuven, Belgium

${ }^{3}$ Professor, Faculty of Psychology and Educational Sciences, KU Leuven, Belgium

*Responding Author

(C) 2016 I TB Vu, K Petry, G Bosmans; licensee IJIP. This is an Open Access Research distributed under the terms of the Creative Commons Attribution License (http://creativecommons.org/licenses/by/2.0), which permits unrestricted use, distribution, and reproduction in any Medium, provided the original work is properly cited.
} 


\section{Peer Victimization and Emotional Problems in Vietnamese Children: A Longitudinal Study}

(Hanish \& Guerra, 2002). However, the effects of peer victimization on emotional problems appear inconsistent across longitudinal studies. Some studies have revealed that children who suffer from victimization in childhood are more likely to experience emotional problems in adolescence (e.g., Zwierzynska et al., 2013; Kumpulainen \& Räsänen, 2000) while other studies have not found evidence to support this link (e.g., Klomek et al., 2008).

Although there are many studies indicating the associations between peer victimization and emotional problems as mentioned above, research conducted in Vietnam on this topic is still limited. Meanwhile, the problem of peer victimization has been on the rise in Vietnamese schools in recent years. More specifically, a study by Hang and Tam (2013) revealed that peer victimization increased rapidly from $8.0 \%$ in 2006 to $19.7 \%$ in 2009 in their research sample. So far, there are several studies that stop at finding only the cross-sectional associations between peer victimization and emotional problems in Vietnam. For example, among students in Vietnamese secondary schools, Huong (2012) and Tran (2013) revealed significant crosssectional relationships between being exposed to some types of bullying with anxiety. However, little is known about the long-term costs of peer victimization in the Vietnamese context. Thus, to address this, the present study will look into the emotional effects of the peer victimization phenomenon on Vietnamese school-aged children. Namely, the current study will dedicate to investigate whether long-term emotional effects can be found among children in Vietnam.

To fully understand the longitudinal link between peer victimization and emotional problems mentioned above, it is necessary to find out which factors contribute to that association. One factor explaining longitudinal effects might be that victims develop emotional problems during the bullying episodes and then retain these emotional problems over time, suggesting that the bullying should be considered an emotional scar from which it is hard to recover. Victims of bulling may develop emotional problems if they have some emotion problems, such as feeling isolated, at the same time of being bullied (e.g., Newman et al., 2005). At the same time, literature has articulated that emotional problems mediate the cross-sectional association and longitudinal link between peer victimization and maladjustments (e.g., Hoglund \& Leadbeater, 2007; Baker \& Bugay, 2011; McLaughlin et al., 2009). Based on these empirical evidences, it is reasonable to suggest that current emotional problems which co-occur with peer victimization may mediate the longitudinal relationship between this peer victimization and later emotional problems. The present research will investigate the mediation role of concurrent emotional problems and the longitudinal links between the peer victimization predictor and the outcome of later emotional problems in Vietnamese children.

In addition to investigating the mediation role of concurrent emotional problems, exploring the role of gender is useful to understanding the association between peer victimization and emotional problems. However, it is necessary to note that there is inconsistency among research conducted outside Vietnam about gender as a factor that moderates both cross-sectional and 
longitudinal associations between peer victimization and emotional problems. For example, in some cross-sectional studies, researchers found that being bullied predicts depression among boys (e.g., Kaltiala-Heino et al., 2010), while others revealed that there is no gender effect in this association (e.g., Rudolph et al., 2011). Similarly, in some longitudinal studies, there is mixed evidence about whether gender significantly moderate the links between the two factors (e.g., Snyder et al, 2003; Goodman et al., 2001). In contrast with the many peer victimization research projects conducted in Western countries which discuss gender roles, there is little research of the role of gender in peer victimization in Vietnam. Utilizing a longitudinal approach, the present study is dedicated to examining the influence of gender on both cross-sectional and longitudinal links between peer victimization and emotional problems among Vietnamese children.

\section{THE CURRENT STUDY}

The present study aimed to investigate the links between peer victimization and emotional problems in an eight-year follow-up study with two waves in Vietnamese children. First, we examined cross-sectional associations between peer victimization and emotional problems at both waves. Second, we determined whether peer victimization at age 8 (peer victimization at wave 1, PV1) would predict emotional problems at age 15 (emotional problems at wave 2, EP2). Moreover, we investigated whether the effect of PV1 on EP2 was mediated by emotional problems at age 8 (emotional problems at wave 1, EP1). Finally, we examined the effect of gender in the associations between peer victimization and emotional problems. Based on our current study and on the existing research corpus, we expected to find that: (1) there are significant cross-sectional associations between peer victimization and emotional problems at both waves; (2) peer victimization significantly predicts emotional problems across time; (3) emotional problems at wave 1 is a mediating factor that significantly mediates the longitudinal link between peer victimization at wave 1 and emotional problems at wave 2 (indirect link). We also wanted to explore whether both cross-sectional and longitudinal relationships are affected by gender. The results from this study would give us a better understanding about the long-term emotional consequences of peer victimization in Vietnam.

\section{METHOD}

To address the aim of the current study, we used data collected in an international study of childhood poverty which is the Young Lives study, involving 12,000 children over fifteen years in four countries, including Vietnam. More specifically, the study followed two cohorts of children in each country during the fifteen-year span. They include 1,000 children born in 2001 or 2002 and 2,000 children born in 1994 or 1995. In Vietnam, the Young Lives study measured the childhood wellbeing of the two cohorts in addition to economic, social, physical and demographic aspects in different geographical areas of the country. The Young Lives study administered a variety of questionnaires covering many topics, including the issue of peer victimization and emotional problems, to the older cohort (Tuan et al., 2003; Thang et al., 2011). The dataset relates to peer victimization and emotional problems was utilized because it fits the 
Peer Victimization and Emotional Problems in Vietnamese Children: A Longitudinal Study

purpose of the current research. The Young Lives dataset is available from the UK Data Service, which we obtained from UK Data Service as a data file named "SN7483" (Boyden, 2014). As a result, we had a database that contains information on older cohort who were evaluated in a seven-year follow-up period, from age 8 (wave 1) to age 15 (wave 2).

\section{Participants}

The participants from the older cohort (born in 1994 or 1995) were followed in two waves. More specifically, there were 1,000 children in total ( $50.2 \%$ boys) in the survey in wave 1 (Tuan et al., 2003; Thang et al., 2011). However, the number reduced to 976 children (49.3\% boys) in wave 2 of the survey. The age of children was 8-years-old in the first wave and 15-years-old in the second wave (Tuan et al., 2003). The primary caregivers reported on the questionnaire about their children in the first wave (97.2\% females). Most of the caregivers who reported on the questionnaire about their children in the first wave (95.4\%) were the children's biological mothers. The rest of the reporters were grandmothers (1.5\%), fathers (2.4\%), aunts/uncles $(0.2 \%)$, and other $(0.2 \%)$. Children reported for themselves on the questionnaire in the second wave. According to the dataset from the Young Lives study (Boyden, 2014), almost all of these children attended formal school (98.8\%) and most of them attended public school (99.9\%). $79.3 \%$ of the children's families were economically comfortable (managed to get by), while $12.2 \%$ of the families were economically disadvantaged (never had quite enough for living). Although Young Lives conducted their Vietnamese study using a largely economically disadvantaged sample, Thang et al. (2011) indicated that their data sample represents a wide socioeconomic variety of Vietnamese children and that their sample can be used as "an appropriate and valuable instrument for analyzing correlates and causal relations, and for modeling child welfare and its longitudinal dynamics in Vietnam” (p. 22).

\section{Measures}

\section{Peer victimization}

At age 8 , peer victimization was measured by only one item: "Picked on or bullied by other children". This item was derived from the Goodman's Strengths and Difficulties Questionnaire, abbreviated SDQ (Tuan et al., 2003) with three response options ranging from Not true, Somewhat true to Certainly true. This individual item belongs to the peer relationship problem subscale of the SDQ and the scale had not been validated in Vietnamese population (Tran et al., 2003) at that time. This item was answered by the children's primary caregivers.

At age 15, nine items developed by Young Lives researchers (Thang et al., 2011) were used to measure peer victimization including: 1) Called you names or sworn at you; 2) Tried to get you into trouble with your friends; 3) Took something without permission or stole things from you; 4) Made fun of you for some reasons; 5) Made you uncomfortable by staring at you for a long time; 6) Punched, kicked or beat you up; 7) Hurt you physically in some way; 8) Tried to break or damaged something of yours; 9) Refused to talk to you or made other people not talk to you.

(C) The International Journal of Indian Psychology, ISSN 2348-5396 (e)| ISSN: 2349-3429 (p) | 146 
These items were rated in a 4-point Likert scale ranging from Never, Once, 2-3 times to 4 or more times. In the current study, Cronbach's Alpha for these nine items was acceptable $(\alpha=$ 0.78). These items were reported by children.

\section{Emotional problems}

Emotional problems at both waves were also measured by the emotional symptoms subscale of the SDQ (Tran et al., 2003). Regarding wave 1, children's main care givers were asked to report on their child's emotional problems by answering the items in the subscale. There were five items in the subscale including: 1) Often complains of headaches, stomach aches or sickness; 2) Many worries, often seems worried; 3) Often unhappy, downhearted or tearful; 4) Nervous or clingy in new situations; and 5) Many fears, easily scared. These items were answered by primary caregivers in a 3-point Likert scale ranging from Not true, Somewhat true to Certainly true. In the current study, Cronbach's Alpha for these items was slightly low $(\alpha=0.63)$.

At age 15, because the respondents changed from primary caregivers to the children themselves, there were some changes in the wording of the SDQ's emotional symptoms subscale. Namely, they are: 1) You worry a lot; 2) You get a lot of headaches, stomach aches or sickness; 3) You are often unhappy, downhearted or tearful; 4) You are nervous in new situations; 5) You have many fears, you are easily scared. Children reported on these items with three options ranging from Not true for me, A little true for me to Certainly true for me. The reliability of this subscale for the present study was slightly slow with Cronbach's alpha coefficients $\alpha=0.67$.

\section{RESULTS}

\section{Descriptive results}

Preliminary descriptive statistics about peer victimization and emotional problems at both waves for the total sample data are presented in Table 1.

Table 1. Descriptive statistics, t-test about peer victimization and emotional problems at both waves.

\begin{tabular}{|l|l|l|l|l|}
\hline \multirow{2}{*}{} & \multirow{2}{*}{ Mean(SD) } & Gender & \multirow{2}{*}{ t-test } \\
\cline { 3 - 4 } & & Mean Males (SD) & Mean Females (SD) & \\
\hline PV1 & $1.91(0.88)$ & $1.90(0.87)$ & $1.91(0.89)$ & 0.06 \\
\hline EP1 & $9.02(2.68$ & $8.88(2.59)$ & $9.17(2.27)$ & 1.69 \\
\hline PV2 & $11.99(3.75)$ & $12.01(3.90)$ & $11.98(3.60$ & -0.15 \\
\hline EP2 & $8.64(2.16)$ & $8.25(2.10)$ & $9.01(2.15)$ & $5.51^{* * *}$ \\
\hline
\end{tabular}

Note. ${ }^{* *} p<.01,{ }^{* * *} p<.001$; PV1 = Peer victimization at wave 1; EP1 = Emotional problems at wave 1; PV2 = Peer Victimization at wave 2; EP2 = Emotional problems at wave 2 


\section{Cross-sectional associations}

We tested correlations between peer victimization and emotional problems at both waves. Results showed that peer victimization positively correlates with emotional problem in two cross-sectional associations at two time points (Table 2).

Table 2. Correlations between peer victimization and emotional problems at both waves

\begin{tabular}{|l|l|l|l|l|l|}
\hline & & PV1 & EP1 & PV2 & EP2 \\
\hline PV1 & 1 & & & \\
\hline EP1 & $.33^{* *}$ & 1 & & \\
\hline PV2 & .01 & .00 & 1 & \\
\hline
\end{tabular}

Note. ${ }^{* *} p<.01,{ }^{* * *} p<.001$; PV1 $=$ Peer victimization at wave 1; EP1 = Emotional problems at wave 1; PV2 = Peer Victimization at wave 2; EP2 = Emotional problems at wave 2

\section{Longitudinal association and mediation effect}

To determine both 1) a longitudinal link between peer victimization and emotional problems and 2) a mediation role of concurrent emotional problems which co-occur at the same time with peer victimization, analysis was made with peer victimization at wave 1 (PV1) as the risk factor, emotional problems at wave 2 (EP2) as the outcome and emotional problems at wave 1 (EP1) as the mediator. Results of the analysis is presented in Figure 1

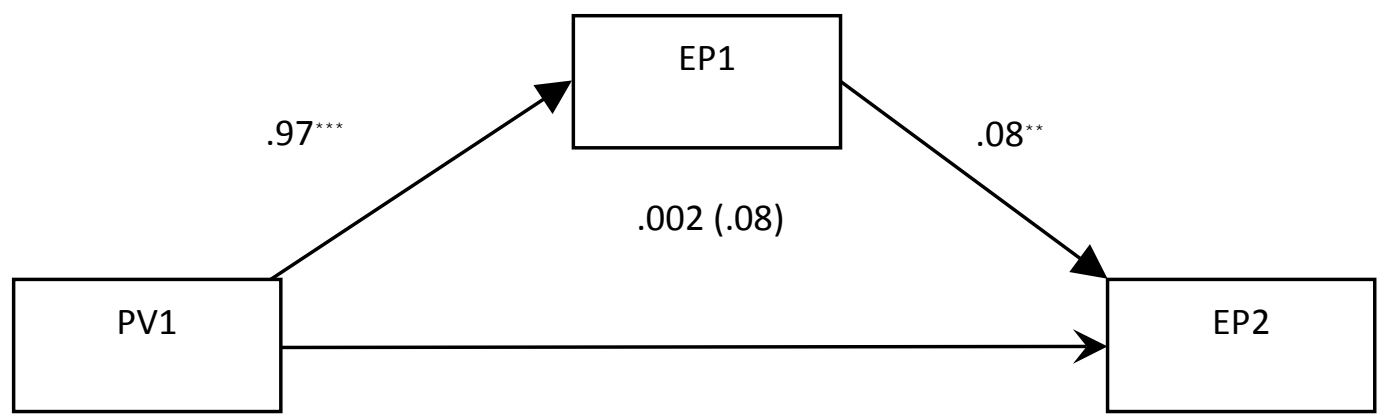

Note. ${ }^{* *} p<.01,{ }^{* *} p<.001$; PV1 = Peer victimization at wave 1; EP1 = Emotional problems at wave 1; EP2 = Emotional problems at wave 2 . The values in the figure reflect unstandardized regression weights.

Figure 1. The final mediation model for emotional problems.

As Figure 1 illustrated, peer victimization at wave 1 did not significantly predict emotional problems at wave $2(\beta=.002, p=.99, n s)$. However, peer victimization was found to positively correlate with emotional problems at wave $1(\beta=.97, p<.001)$ which, in turn, was positively predicted emotional problems at wave $2(\beta=.08, p<.01)$.

In the full mediation model, the relationship between current peer victimization at wave 1 and emotional problems at wave 2 was mediated by emotional problems at wave 1 . The 
unstandardized indirect effect was .08 (.97 x .08). Bootstrapping procedures were used to test the significance of this indirect effect. Unstandardized indirect effects were calculated for each of 10,000 bootstrapped samples. The bootstrapped unstandardized indirect effect was .08 with 95\% confidence interval ranging from .03 to .13. As a result, emotional problems at wave 1 significantly mediated the longitudinal link between peer victimization at wave 1 and emotional problems at wave 2 .

\section{Gender effects}

We examined the moderator role of gender in cross-sectional associations between peer victimization and emotional problems at both waves. Although there was a significant gender difference in emotional problems seen in wave 2 (Table 1), gender did not significantly moderate the association between peer victimization and emotional problems at wave $1(p=.09$, ns) and wave $2(p=.30, n s)$. Gender was also found not to moderate the longitudinal link between peer victimization at wave 1 and emotional problems at wave $2,(p=.23, n s)$.

\section{DISCUSSION}

This current study aimed to investigate the long-term cost of peer victimization on emotional problems in Vietnamese children. We hypothesized that there were significant associations between peer victimization and emotional problems both between and within waves. In addition, we expected that emotional problems that co-occur with peer victimization would significantly mediate the longitudinal link between peer victimization and later emotional problems. We also predicted that gender would moderate the associations between peer victimization and emotional problems at both cross-sectional relationships and longitudinal link. To test these hypotheses, we used bivariate correlation, mediation and moderation analysis in the current study. Some hypotheses have been confirmed in the present study. Namely, the results from the current study confirm that peer victimization and emotional problems positively correlated at both waves. However, we found no evidence to support the longitudinal link between peer victimization and emotional problems as hypothesized in the current study, as current peer victimization did not significantly predict emotional problems over time. In line with our predictions, current emotional problems were found to significantly mediate the longitudinal link between current peer victimization and later emotional problems. With regard to gender effect, no significant evidence was found to confirm the hypothesis that gender significant moderated the associations between peer victimization and emotional problems at both cross-sectional relationships and longitudinal link. One possible explanation for these results is that both sexes experienced peer victimization and emotional problems equally in most cases except for emotional problems at wave 2.

The cross-sectional correlations between peer victimization and emotional problems in the present study align with the literature indicating that peer victimization is associated with emotional problems (e.g., Hawker \& Boulton, 2000). These results are also consistent with 
Peer Victimization and Emotional Problems in Vietnamese Children: A Longitudinal Study

Vietnamese studies (e.g., Tran, 2013; Huong et al., 2012). However, this study differed from the studies of Tran (2013) and Huong et al. (2012) in that we considered the cross-sectional associations between peer victimization and emotional problems in a larger population of children over different cohorts, from middle childhood (age 8) to early adolescence (age 15). Our findings provide empirical evidence to support the cross-sectional association of peer victimization and emotional problems throughout the course of development among Vietnamese children.

While peer victimization positively correlated with emotional problems in both cross-sectional relationships, it did not significantly predict emotional problems seven years later. This finding is in line with those of Snyder et al. (2003), and Malti et al. (2010), which revealed that peer victimization does not predict emotional symptoms in children in the long-term. However, Snyder et al. (2003) and Malti et al. (2010) reported that increases in peer victimization predicts emotional symptoms in boys. One reason why we could not replicate the latter effect, might have been the use of different informants between the two waves: student's main caregivers as opposed to students themselves, which typically do not highly correlate (e.g., Lewis et al., 2014; Løhre et al., 2011). In addition, differences in the measurement of peer victimization at the two waves also make it difficult to examine this hypothesis. Finally, the long time between the current study's measurement waves might have resulted in low correlations.

Although these results suggest that peer victimization does not have a significant long-term impact on children's emotional development, the current study's mediation analysis warrants caution for such conclusions. More specifically, the emotional problems children experience in association with peer victimization significantly mediate the longitudinal relationship between the two factors. That is, peer victimization positively correlated to later emotional problems through the mediating role of concurrent emotional problems. This finding is consistent with prior research indicating emotional problems as risk factors that mediate the association between peer victimization and emotional maladjustment (e.g., McLaughlin et al., 2009; Baker \& Bugay, 2011). Consequently, it can be concluded that concurrent emotional problems were risk factors that increase later emotional problems among children in Vietnam who have been bullied. As a result, the current study contributes to the literature on the role of the social-cognitive process in mediating the link between peer victimization and emotional adjustment problems in children (Crick \& Dodge, 1994; Hoglund \& Leadbeater, 2007) through replicating it in a sample of Vietnamese children. Namely, the emotional problems which are part of social-cognitive process deeply affect children's emotional adjustment (Crick \& Dodge, 1994).

The final purpose of this study was to examine the effects of gender on the cross-sectional relationships as well as the longitudinal association between peer victimization and emotional problems. Although girls were found to report more emotional problems than boys at wave 2, gender was not a significant moderator of the associations between peer victimization and 
Peer Victimization and Emotional Problems in Vietnamese Children: A Longitudinal Study

emotional problems on both the cross-sectional relationships and longitudinal link. These findings add to the current body of evidence that finds no gender effect on the association between peer victimization and emotional problems. This suggests that the emotional cost of being bullied is comparable for boys and girls. This finding is important because it would mean that interventions addressing the long-term cost of peer victimization on consequent emotional problems should equally concentrate on both genders in Vietnam.

\section{CONTRIBUTIONS AND LIMITATIONS}

A strength of this paper is its relatively large sample size which allows results to be representative of Vietnamese children. In addition, this study revealed that concurrent emotional problems are the factors that increase the risk of additional emotional problems among children bullied in Vietnam. This finding may be helpful for seeking interventions that address the longterm effect of peer victimization by focusing on treatment of emotional problems for peer victimized children.

Although the current study has several notable strengths which include the longitudinal approach in a relatively large sample size, it is not without limitations. These limitations relate to the reliability and validation of the research questionnaires. With regard to reliability, peer victimization at wave 1 was only measured by one item. Meanwhile, literature expressed concerns that a single item measure provides inadequate information to estimate measurement properties (McIver \& Carmines, 1981).

With regard to validity, the scale used to measure peer victimization and emotional problems at wave 1 had not been validated at the time that the survey was conducted with the Vietnamese population (Tran et al., 2003). In addition, regarding the scale utilized to measure the two factors at wave 2, no information related to their validity has been found so far. Therefore, it is necessary to conduct further longitudinal studies which use scales featuring good psychometric properties to investigate the association between the two factors. This will help provide more sufficient evidence about the long-term cost of peer victimization in Vietnamese school settings.

Another limitation is the difference of the informants at each time point. More specifically, the children's primary caregivers were the respondents in the first wave while the children filled that role in the second wave. Although there is evidence that reports from different informants have equivalent validity in assessing childhood bullying (Shakoor et al., 2011), this limitation made it difficult to compare peer victimization as well as emotional problems at wave 1 with those at wave 2. Nevertheless, using multiple informants and still finding significant effects over such a long time period are serious indicators that these results are underestimations rather than overestimations of the actual effect size. In other words, these findings should be considered as highly valuable in the debate on the long-lasting emotional damage children experience as a result from being victimized by peers. Moreover, these findings should be an incentive to 
increase efforts to reduce peer victimization and to help its victims, not only in Vietnam, but also globally.

\section{CONCLUSION}

The current study examined the long-term cost of peer victimization on emotional problems in a longitudinal study about Vietnamese children. It revealed that peer victimization and emotional problems in the Vietnamese context are two parallel problems during the course of development from childhood to middle adolescence. In addition, current emotional problems were found to be significant risk factors that cause peer victimization to have a significant impact on future emotional problems. These findings indicate the need for additional studies. First, because the social-cognitive process links peer victimization to emotional problems, it would be interesting to conduct studies focusing on the effects of peer victimization on social-cognitive process and how this process influences behavioral tendency among Vietnamese children. Second, findings on the role of emotional problems suggest the need to implement research which focuses on developing strategies for treating emotional problems in Vietnamese children over the course of childhood development.

\section{Acknowledgments}

The author appreciates all those who participated in the study and helped to facilitate the research process.

\section{Conflict of Interests}

The author declared no conflict of interests.

\section{REFERENCES}

Baker, O. E., \& Bugay, A. (2011). Peer victimization and depressive symptoms: The mediation role of loneliness. Procedia-Social and Behavioral Sciences, 30, 1303-1307.

Boyden, J., Young Lives: an International Study of Childhood Poverty: Rounds 1-3 Constructed Files, 2002-2009 [computer file]. Colchester, Essex: UK Data Archive [distributor], April 2014. SN: 7483, http://dx.doi.org/10.5255/UKDA-SN-7483-1.

Crick, N. R., \& Dodge, K. A. (1994). A review and reformulation of social informationprocessing mechanisms in children's social adjustment. Psychological bulletin, 115(1), 74.

Goodman, M. R., Stormshak, E. A. \& Dishion, T. J. (2001). The significance of peer victimization at two points in development. Applied Developmental Psychology22, 507-526

Hang, N. T. T., \& Tam, T. N. T. M. (2013). School Violence Evidence from Young Lives in Vietnam. Vietnam Policy Paper, 1.

Hanish, L. D., \& Guerra, N. G. (2002). A longitudinal analysis of patterns of adjustment following peer victimization. Development and psychopathology, 14(01), 69-89. 


\section{Peer Victimization and Emotional Problems in Vietnamese Children: A Longitudinal Study}

Hawker, D. S., \& Boulton, M. J. (2000). Twenty years' research on peer victimization and psychosocial maladjustment: a meta-analytic review of cross-sectional studies. Journal of child psychology and psychiatry, 41(4), 441-455.

Hoglund, W. L., \& Leadbeater, B. J. (2007). Managing Threat: Do Social-Cognitive Processes Mediate the Link Between Peer Victimization and Adjustment Problems in Early Adolescence?. Journal of Research on Adolescence, 17(3), 525-540.

Huong, N. T., Tien, T. Q., Chi, H. K., Anh, N. Q., \& Phuong, N. H. (2012). Some mental health problems and influential factors of secondary school students in Hanoi, Vietnam. Greater Mekong Sub-region Initiative e-Journal of Public Health (GMSI-eJPH), 1(2).

Kaltiala-Heino, R., Fröjd, S., \& Marttunen, M. (2010). Involvement in bullying and depression in a 2-year follow-up in middle adolescence. European Child \& Adolescent Psychiatry, 19(1), 45-55.

Klomek, A. B., Sourander, A., Kumpulainen, K., Piha, J., Tamminen, T., Moilanen, I., ... \& Gould, M. S. (2008). Childhood bullying as a risk for later depression and suicidal ideation among Finnish males. Journal of affective disorders, 109(1), 47-55.

Kochenderfer-Ladd, B. (2004). Peer victimization: The role of emotions in adaptive and maladaptive coping. Social Development, 13(3), 329-349.

Kumpulainen, K., \& Räsänen, E. (2000). Children involved in bullying at elementary school age: their psychiatric symptoms and deviance in adolescence: an epidemiological sample. Child abuse \& neglect, 24(12), 1567-1577.

Lewis, A. J., Bertino, M. D., Bailey, C. M., Skewes, J., Lubman, D. I., \& Toumbourou, J. W. (2014). Depression and suicidal behavior in adolescents: a multi-informant and multimethods approach to diagnostic classification. Frontiers in psychology, 5.

Løhre, A., Lydersen, S., Paulsen, B., Mæhle, M., \& Vatten, L. J. (2011). Peer victimization as reported by children, teachers, and parents in relation to children's health symptoms. BMC Public Health, 11(1), 1.

Malti, T., Perren, S., \& Buchmann, M. (2010). Children's peer victimization, empathy, and emotional symptoms. Child Psychiatry \& Human Development, 41(1), 98-113.

McIver, J., \& Carmines, E. G. (1981). Unidimensional scaling (Vol. 24). Sage.

McLaughlin K. A., Hatzenbuehler M. L., Hilt L. M. (2009). Emotion dysregulation as a mechanism linking peer victimization to internalizing symptoms in adolescents. Journal of Consulting and Clinical Psychology, Vol 77(5), 894-904

Newman, M. L., Holden, G. W., \& Delville, Y. (2005). Isolation and the stress of being bullied. Journal of adolescence, 28(3), 343-357.

Nguyen, D. T., Dedding, C., Pham, T. T., Wright, P., \& Bunders, J. (2013b). Depression, anxiety, and suicidal ideation among Vietnamese secondary school students and proposed solutions: a cross-sectional study. BMC public health, 13(1), 1195.

Reijntjes, A., Kamphuis, J. H., Prinzie, P., \& Telch, M. J. (2010). Peer victimization and internalizing problems in children: A meta-analysis of longitudinal studies. Child abuse \& neglect, 34(4), 244-252. 
Rudolph K. D, Troop-Gordon W., Schmidt J. D., \& Helssel E. T., (2011). A Latent Growth Curve Analysis of Early and Increasing Peer Victimization as Predictors of Mental Health across Elementary School. Journal of Clinical Child \& Adolescent Psychology, 40(1), 111-122, 2011

Shakoor, S., Jaffee, S. R., Andreou, P., Bowes, L., Ambler, A. P., Caspi, A., ... \& Arseneault, L. (2011). Mothers and children as informants of bullying victimization: results from an epidemiological cohort of children. Journal of abnormal child psychology, 39(3), 379-387.

Snyder, J., Brooker, M., Patrick, M. R., Snyder, A., Schrepferman, L., \& Stoolmiller, M. (2003). Observed peer victimization during early elementary school: Continuity, growth, and relation to risk for child antisocial and depressive behavior. Child Development, 74(6), 1881-1898.

Thang, N., Van Tien, N., Hang, M. T., \& Thuy, V. T. T. (2011). How Do Children Fare in the New Millennium?

Tran, C. V. (2013). Relations Between Peer Victimization, Self-cognitions and Depression in the United States and Vietnam (Doctoral dissertation, Vanderbilt University).

Tuan, T., Lan, P. T., Harpham, T., Huong, N. T., Thach, T. D., Tod, B., ... \& Ha, N. T. V. (2003). Young Lives Preliminary Country Report: Vietnam.

Weiss, B., Dang, M., Trung, L., Nguyen, M. C., Thuy, N. T. H., \& Pollack, A. (2014). A nationally representative epidemiological and risk factor assessment of child mental health in Vietnam. International perspectives in psychology: research, practice, consultation, 3(3), 139.

Zwierzynska, K., Wolke, D., \& Lereya, T. S. (2013). Peer victimization in childhood and internalizing problems in adolescence: a prospective longitudinal study. Journal of abnormal child psychology, 41(2), 309-323.

How to cite this article: TB Vu, K Petry, G Bosmans (2016), Peer Victimization and Emotional Problems in Vietnamese Children: A Longitudinal Study, International Journal of Indian Psychology, Volume 3, Issue 4, No. 58, ISSN 2348-5396 (e), ISSN: 2349-3429 (p), DIP: 18.01.056/20160304, ISBN: 978-1-365-24976-1

(C) The International Journal of Indian Psychology, ISSN 2348-5396 (e)| ISSN: 2349-3429 (p) | 154 